\title{
Importance of Dental Sleep Medicine as an Integral Part of Dental Curriculum
}

\author{
Nasser Alqahtani, BDS, MS"; Turki Alajaji, BDS²; Raed Alasim, BDS²; Omar Alzoman, BDS²; Hanan Alotaibi, BDS, MS³; Sahar \\ Albarakati, BDS, $\mathrm{MS}^{1} ;$ Waled Alshhrani, $\mathrm{BDS}, \mathrm{MS}, \mathrm{PhD}^{3}$
}

${ }^{1}$ Pediatric Dentistry and Orthodontics Department, College of Dentistry, King Saud University, Riyadh, Saudi Arabia, ${ }^{2}$ General dentist in private clinic, Riyadh, Saudi Arabia; ${ }^{3}$ Prosthetic Dental Science Department, College of Dentistry, King Saud University, Riyadh, Saudi Arabia

\begin{abstract}
Background: Dental sleep medicine (DSM) is a multidisciplinary specialty requiring the combined efforts of physicians in the diagnosis of obstructive sleep apnea (OSA) and dental practitioners in fabricating oral appliance therapy (OAT) in patients, who are contraindicated for positive airway pressure treatment. The comprehensive management of OSA involves the expertise of dental specialists of oral medicine, oral and maxillofacial surgery, orthodontics, and orofacial pain dysfunction fraternities. DSM-trained and -certified dentists, in collaboration with physicians, can aid in reducing the public health burden of undiagnosed and untreated sleep-related breathing disorders. However, very few dentists practice DSM in Saudi Arabia, which may be because of the insufficient teaching hours dedicated to this type of education.

Methods: This descriptive and cross-sectional study examined Saudi dental schools' curricula during the 2017-2018 academic year. Through an electronic survey we determined the number of dental schools that offer DSM training and the average number of teaching hours in DSM education.

Results: Twenty of the 23 dental schools that received the survey responded. Of those, $80 \%$ (16 of 20) reported minimal teaching hours of DSM training. The average number of teaching hours devoted to sleep disorders in Saudi bachelor dental programs was $1.95 \mathrm{~h}( \pm 2.06)$. Teaching hours were reported most often in the fifth academic year, with an average of $0.8 \mathrm{~h}( \pm 0.62)$. There is apparent consensus among the deans of the participatory institutions that DSM education is only didactic in nature. Sixty-five percent of the responding schools covered OSA in their curricula, and $60 \%$ covered sleep bruxism.
\end{abstract}

Keywords: cross-sectional, dental curriculum, dental sleep medicine, electronic survey

Citation: Alqahtani N, Alajajib T, Alasimc R, Alzomand O, Alotaibie H, Albarakati S, Alshhrani W. Importance of dental sleep medicine as an integral part of dental curriculum. J Dent Sleep Med. 2020;7(4)

\section{INTRODUCTION}

Obstructive sleep apnea (OSA) ${ }^{1}$ is the most common type of sleep-disordered breathing (SDB). ${ }^{2}$ It is characterized by recurrent episodes of obstruction of the upper airway, resulting in sleep fragmentation and oxygen desaturation. OSA has become a major public health issue associated with serious consequences, including hypertension, arrhythmia, stroke, and heart failure. ${ }^{3}$ Moreover, sleep fragmentation and recurrent hypoxemia causes daytime sleepiness and impaired concentration, which increases the risk of motor vehicle collisions and workplace accidents. ${ }^{4}$

Dental education enables practitioners to exhibit professionalism in future clinical practice. ${ }^{5,6}$ Designing a well-structured dental curriculum will ensure the best learning outcomes. ${ }^{7,8}$ Dental schools all over the world have a common objective of helping their students to graduate as highly qualified dentists. However, the curricula, methods of teaching, years of study, and the caliber of the dental graduates vary at national and international levels. Benchmarking usually helps prepare future clinicians to face demanding situations such as SDB. ${ }^{9,10}$ Wide variation is evident in the prevalence of SDB among different populations. ${ }^{11}$ In the United States, the prevalence of OSA has increased by $12 \%$ in the adult population, ${ }^{12}$ but only $20 \%$ of those cases are diagnosed. It has been estimated that in North America, 1 in 4 middleaged men and 1 in 10 middle-aged women have OSA. ${ }^{1}$ In Saudi Arabia, two studies estimated that 3 of 10 Saudi men and 4 of 10 Saudi women are at high risk of the development of OSA. ${ }^{13,14}$

Despite the great progress made in sleep medicine, only a few dentists in Saudi Arabia have the required training in the management of OSA. ${ }^{9,15}$ This area of dentistry is called dental sleep medicine (DSM), and it focuses on modalities to manage SDB. ${ }^{16}$ DSM focuses on the use of oral appliance therapy (OAT) to treat SDB, including obstructive sleep apnea (OSA) and snoring. ${ }^{16}$ Recently, DSM was recognized as a discipline concerned with the study of the oral and maxillofacial causes and consequences of sleep-related problems. ${ }^{17}$

Dental specialists, when trained in DSM, can help physicians identify and effectively manage sleep-related bruxism, primary snoring, and OSA. ${ }^{13}$ Specifically, as part of routine dental examinations, dentists can play a significant role in detecting OSA, make appropriate recommendations and referrals for patients with OSA, and treat OSA with oral appliances. ${ }^{18}$ However, dentists' involvement in OSA treatment still appears unsatisfactory 
in Saudi Arabia and this may be because of insufficient DSM educational hours in bachelor dental programs. The purpose of this study was to assess the hours of DSM education included in the curricula of Saudi dental colleges.

\section{MATERIALS AND METHODS}

A cross-sectional observational study was carried out during the 2017-2018 academic year. An electronic survey (questionnaire) was sent to 23 dental schools in Saudi Arabia. The questionnaire used in this study was adopted from a predesigned questionnaire intended to gather information about the teaching of DSM topics. ${ }^{19}$ Assuming a statistical power of $80 \%$ with $95 \%$ confidence level based on similar studies done in other geographic domains, 23 of the 24 dental schools in Saudi Arabia consented to participate in the study. The questionnaire, which consisted of nine main questions, was created using Google Forms and emailed to the deans and vice deans of 23 dental colleges in Saudi Arabia. The questionnaire comprised questions intended to acquire data to assess the number of dental schools that offer DSM training, the year of learning, and the average number of teaching hours in DSM education. In addition, questions testing the prevailing knowledge about the available treatment modalities for OSA and their implications were also included in the questionnaire. The collected data were tabulated, anonymizing the dental school with numerals and using assigned variable names for the questions Q1 to Q9, and statistically analyzed to evaluate the desired outcome variables.

Descriptive statistics analysis was performed using $\mathrm{R}$ statistical software (version 3.3.1; R Foundation for Statistical Computing, Vienna, Austria), and graphs were produced using GraphPad Prism V.8 for Mac OS X (GraphPad Software, San Diego, California, USA).

\section{RESULTS}

Only 20 schools (87\%) responded because 3 of the colleges were new and did not yet have a graduate class. Of the dental schools that responded, $16(80 \%)$ reported that their curricula included only a few DSM topics. The mean number of teaching hours devoted to sleep disorders in the Saudi Bachelor of Dental Science Surgery programs was $1.95 \mathrm{~h}( \pm 2.06)$, with a range of 0 to 8 hours and a mode of 1 hour (Figure 1). These teaching hours were most often reported in the fifth academic year, with an average of 0.8 $\mathrm{h}( \pm 0.62)$, followed by the fourth year, with a mean of 0.6 h ( \pm 0.99$)$ (Figure 2).

Fifteen schools reported that students had a DSM curriculum with completely didactic learning experiences. One school offered students hands-on preclinical laboratory experience and observational clinical experiences in addition to the didactic learning. In most of
Figure 1. Number of teaching hours devoted to dental sleep medicine at the dental schools

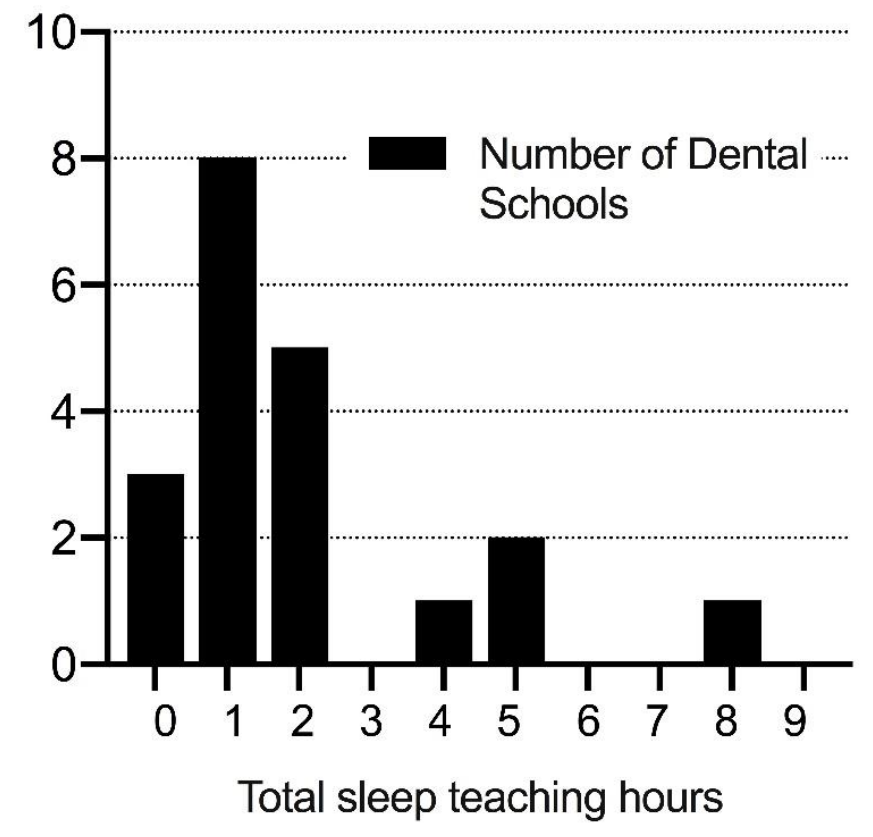

Figure 2. Number of teaching hours devoted to dental sleep medicine in each academic year

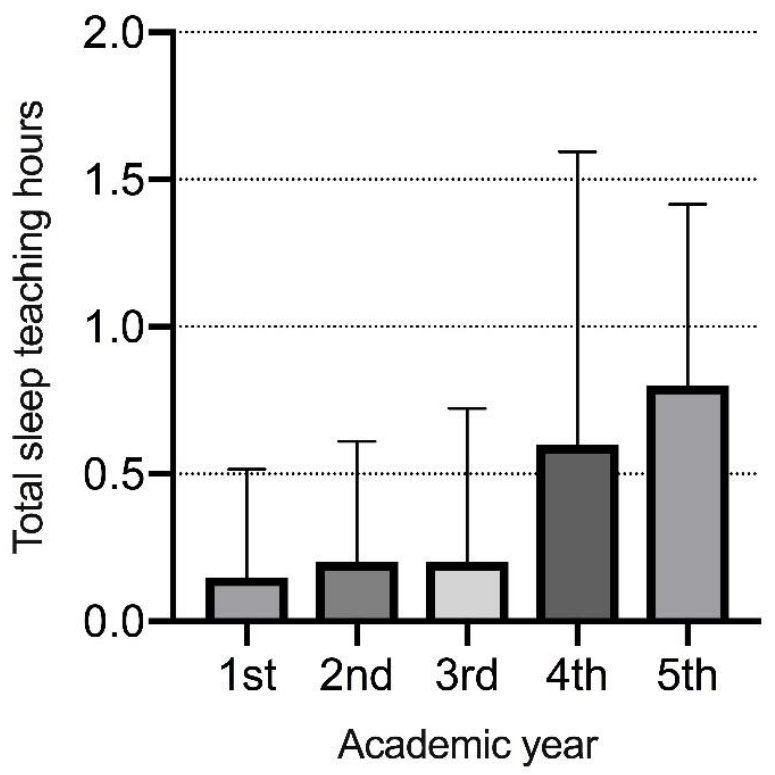

the dental schools (59\%), DSM topics were taught in two or more dental departments, whereas $41 \%$ of the schools reported teaching these topics in only one dental department. The oral medicine and oral surgery departments were most commonly reported $(8$ schools, $40 \%$ ), followed by the prosthodontics and orthodontics departments (6 schools, 30\%) (Figure 3).

A wide spectrum of sleep-related topics and diagnoses were analyzed (Figure 4). Sixty-five percent of the responding dental schools covered OSA in their curricula, 
Figure 3. Number of responding dental schools that reported dental sleep medicine in different departments

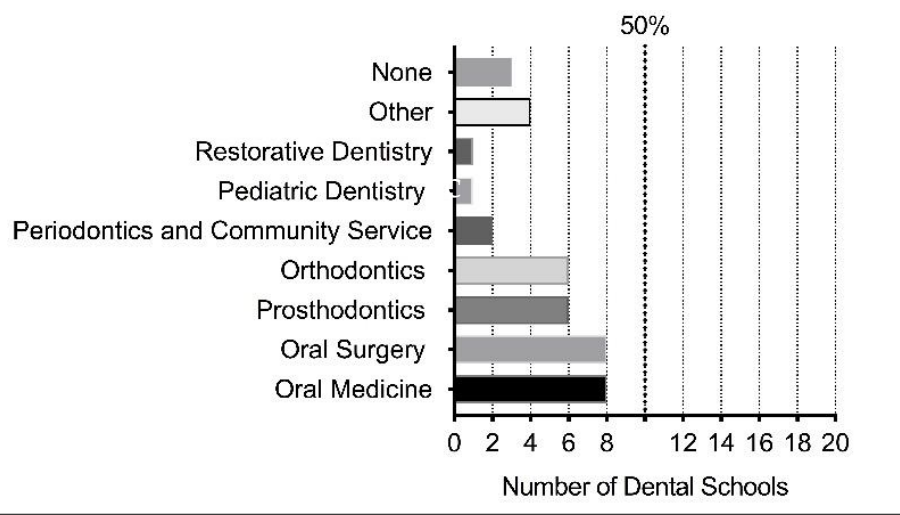

Figure 4. Number of responding dental schools that included different dental sleep medicine topics in their curricula

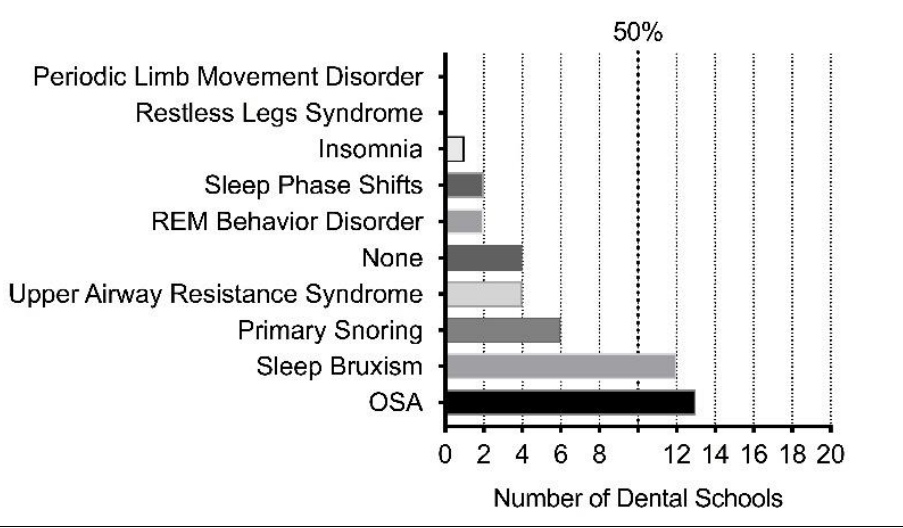

and $60 \%$ covered sleep bruxism. Curiously, snoring, which is often a symptom of OSA, was addressed in only $30 \%$ of the dental schools' curricula.

With regard to the topics regarding SDB treatment and management, $60 \%$ of the responding schools discussed therapeutic interventions with OAT, and $45 \%$ discussed orthodontics approaches. Continuous positive airway pressure (CPAP) was reported by only 5 schools (25\%), although it is undoubtedly considered the standard of care for OSA. Figure 5 summarizes the percentage of responding schools that discussed different therapies for SDB, and Figure 6 summarizes the percentage of responding schools that discussed various aspects of OAT for SDB in their curricula. Figure 7 summarizes the percentage of responding schools that addressed contemporary topics in the DSM field. All 16 responding schools discussed the medical consequences of untreated SDB, but only $25 \%$ discussed the psychosocial consequences. Coordinated care with sleep physicians, polysomnography, and home sleep apnea testing were covered by $30 \%, 20 \%$, and $15 \%$ of the schools, respectively.

Because a low number of teaching hours concerning DSM topics in undergraduate dental curricula were
Figure 5. Number of responding dental schools that included different therapies for sleep-disordered breathing in their curricula

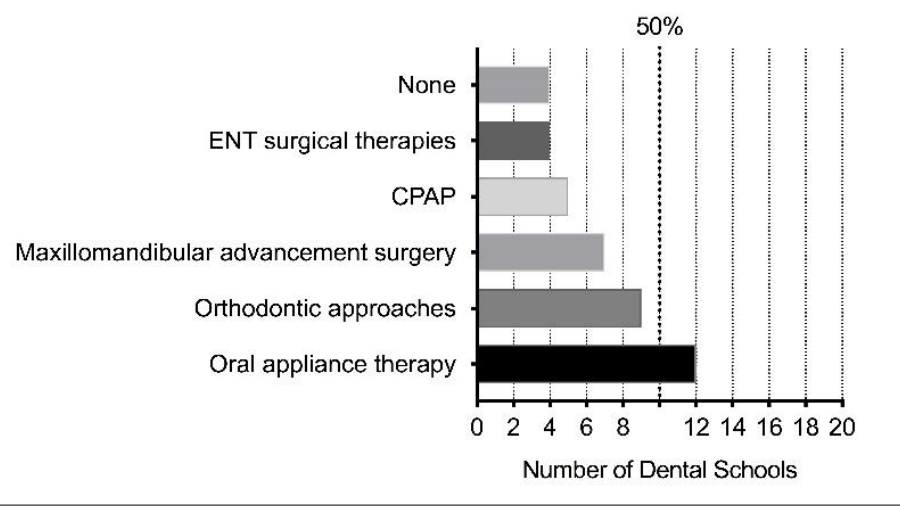

Figure 6. Number of responding dental schools that included different aspects of oral appliance therapy for obstructive sleep apnea in their curricula

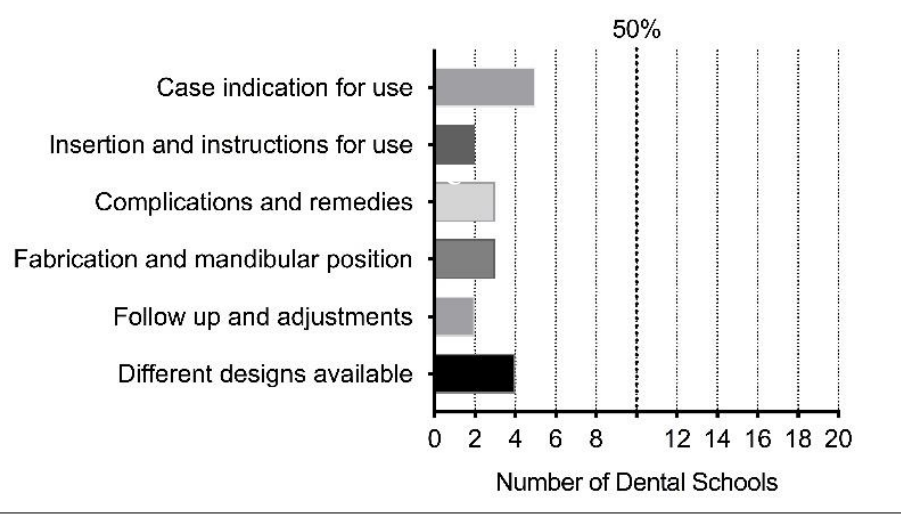

Figure 7. Number of responding dental schools that include different contemporary dental sleep medicine topics in their curricula

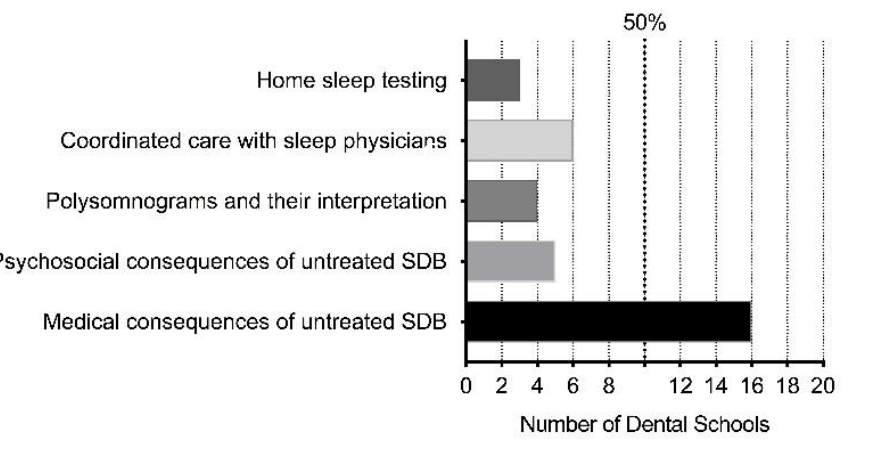

anticipated, the justification for this was explored. The results evaluating the reasons for minimal DSM hours in the institutions are summarized in Figure 8.

\section{DISCUSSION}

Sleep disorders are a major public health problem that affects morbidity and mortality. It has been reported that one-third of the Saudi population is at high risk of the 
Figure 8. Justifications for the low number of teaching hours devoted to sleep disorder topics

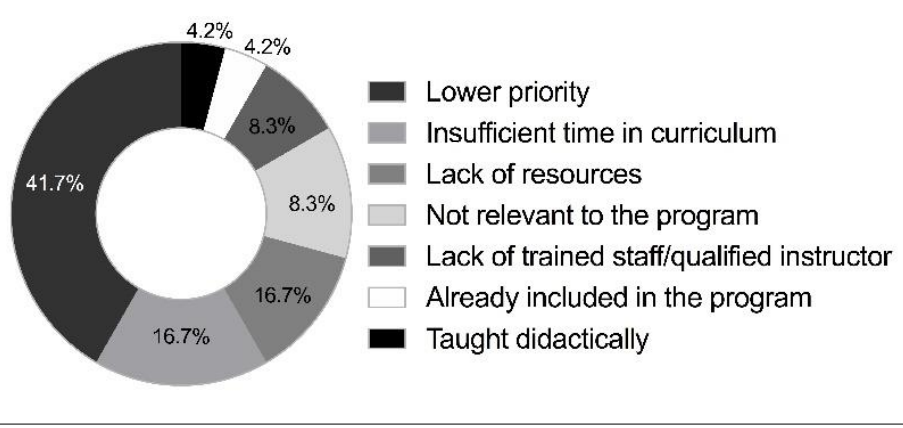

development of OSA, ${ }^{14}$ and a recent study reported that the current prevalence of OSA in the Saudi population is $12.8 \%$ among men and $5.1 \%$ among women. ${ }^{20}$ Thus, the demand for sleep medicine, including DSM, in Saudi Arabia is expected to increase. However, few studies have examined the status of sleep medicine practice in Saudi Arabia, ${ }^{21-23}$ and currently none have discussed the status of DSM practice or education in Saudi Arabia.

The findings of the current study suggest the lack of effective DSM education for dentists during their bachelor dental programs. The current study found that the average number of teaching hours devoted to sleep medicine among bachelor dental programs was 1.95 hours among all the responding Saudi dental schools and 2.25 hours when schools with zero educational hours were excluded. ${ }^{19}$ This result demonstrates that DSM content needs to be increased in Saudi dental curricula.

Previously, this issue was recognized and studied in the United States, ${ }^{19}$ Australia, and New Zealand, ${ }^{19}$ and these studies also found insufficient education on sleep disorders in pre- doctoral dental programs. The first study, which examined US dental schools in the 2008-2009 academic year, reported that the average number of sleep medicine teaching hours in US dental schools was $2.96 \mathrm{~h}$ (and $3.92 \mathrm{~h}$ when schools with zero educational hours were excluded). The second study, which was conducted among dental schools in Australia and New Zealand in the 2011 academic year, reported a better average of predoctoral sleep medicine teaching hours: 4.5 hours. ${ }^{19}$ Similarly, in a previous study conducted in 2012-2013, to assess the undergraduate sleep medicine education among dental students in Middle Eastern countries, the average hours of DSM training was as low as 1.2 hours. ${ }^{24}$ However, the current average teaching hours devoted to DSM in Saudi dental schools (1.9 hours) although showing positive results, still elicits marginal improvement when compared to the DSM teaching hours in the United States, Australia, and New Zealand.

The American Academy of Dental Sleep Medicine conducts a comprehensive 65 -hour mastery program. ${ }^{1}$ Emphasizing the need to provide OAT to patients with OSA and snoring, the program provides dentists with the necessary training and skills needed. ${ }^{25}$ With students being the important stakeholder of any dental program, the curriculum should equip them with interdisciplinary management skills necessary for a graduate dentist. ${ }^{15}$ Future dental practitioners aspiring to specialize in DSM will largely benefit ${ }^{26}$ when they are given preliminary exposure to the diagnostic intricacies and the current treatment modalities available to treat OSA. This is more likely achievable by revamping the current dental curricula and increasing the DSM teaching hours in comparison with the growing demands for sophistication. ${ }^{23}$ In 2013, the first national dental educators' conference, Sleep Disordered Breathing in Dental School Education: Past, Present, and Future $^{19}$ reiterated the importance of providing education on SDB in predoctoral and advanced dental education.

A study on OSA education and knowledge among medical schools in Saudi Arabia reported that more than $80 \%$ of medical students rated their knowledge of OSA as below average, and the teaching hours devoted to sleep medicine topics ranged from 0 to 8 hours, with a mean of $2.6 \pm 2.6$ hours. ${ }^{19}$ Another study reported that Saudi primary care physicians' knowledge of sleep medicine is generally low. ${ }^{27}$ The limited time spent teaching sleep medicine at medical schools in Saudi Arabia might lead to some challenges regarding dentists' involvement in OSA management, as physicians often call on dentists to collaboratively treat patients with SDB and play a vital role in managing this potentially life-threatening sleep disorder. 23-26, 28, 29

Most teaching hours were devoted to DSM only in the fifth academic year (the last year of the program), and purely didactic experiences were provided by all but one school, which also reported only observational clinical experiences. These findings are similar to those of other studies, ${ }^{19,30}$ which reported the maximum number of teaching hours devoted to DSM was in the last year of predoctoral programs. More clinical sleep medicine experience was reported in dental schools in the United States, Australia, and New Zealand; it was reported that 4 dental schools in the United States ( $8 \%$ of the sample) provided hands-on clinical laboratory experience ${ }^{19}$ and that 4 dental schools in Australia/New Zealand $(40 \%$ of the sample) provided clinical experience. ${ }^{19}$ Based on the overall low number of DSM teaching hours found in our study, it seems that clinical DSM experience is not a substantial part of Saudi dental schools' curricula.

The current study also found that the oral medicine and oral surgery departments in eight schools and the prosthodontics and orthodontics departments in six schools taught DSM. Similarly, the oral medicine departments in dental schools in Australia and New Zealand were most frequently involved in teaching sleep medicine. ${ }^{19}$ Among the responding dental schools in this study, the most commonly reported sleep medicine topic was OSA, followed by sleep bruxism. Information about these topics was mainly provided in didactic settings. Moreover, the 
study findings demonstrate that topics about OAT and orthodontic therapy for OSA are the most commonly reported methods of treatment for OSA. CPAP, which is a standard of care treatment for OSA, was reported by only 5 of the responding schools $(25 \%)$. In the United States, CPAP was reported by 37 dental schools $(75.7 \%$ of the sample), whereas OAT was reported by $42(86.5 \%$ of the sample). ${ }^{19}$

Despite the abundant literature indicating that oral appliances are an effective treatment for OSA in adults, ${ }^{31-}$ 33 few schools in this study reported that they educated students about the steps and details of OSA therapy involving such appliances. In fact, almost $75 \%$ of the responding dental schools did not include details about OAT for OSA in their curricula. The administrators of the Saudi dental schools included in the current study reported some justifications for the low number of hours spent teaching DSM topics. The most commonly reported reasons were, surprisingly, "low priority" $(41.7 \%$ of schools) and "insufficient time in curricula" (16.7\% of schools), even though DSM deals with the treatment and management of a potentially life-threatening sleep disorder. Other schools" administrators identified "lack of resources," "not relevant to the program," and "lack of trained staff" as challenges regarding the inclusion of DSM in their curricula. The authors of this article think that it is necessary to develop a defined strategy for the implementation of DSM education in Saudi dental schools' curricula since current dental students' exposure to DSM is fragmented.

\section{CONCLUSION}

Currently, Saudi bachelor dental schools do not meet their students' educational needs with respect to DSM. Within limitations of the study, the survey was completed by at least one faculty member within the dean's office. Further, postdoctoral and specialty training programs were not included in this study. In future, dedicate faculty resources and clinical space to enable appropriate didactic and clinical education on DSM is recommended for dental students and recruit faculty trained in DSM. In addition, faculty and students must be encouraged to explore research opportunities related to DSM. DSM may also be included in the Saudi dental licensure examination. An effective inclusion of DSM not only in the didactic but also the clinical modules of the undergraduate dental education programs will enhance the knowledge and contribution of the dental fraternity in the management of SDB.

\section{ACKNOWLEDGMENTS}

The authors extend their appreciation to the Deanship of Scientific Research at King Saud University for funding this work through Research Group no. RG-1439-54.

\section{REFERENCES}

1. Abdelsattar ZM, Hendren S, Wong SL, Campbell DA Jr, Ramachandran SK. The impact of untreated obstructive sleep apnea oncardiopulmonary complications in general and vascular surgery: a cohort study. Sleep. 2015;38(8):1205-1210.

2. $\quad$ Phillipson EA. Sleep apnea--a major public health problem. $N$ Engl $J$ Med. 1993;328(17):1271-1273.

3. Rodsutti J, Hensley M, Thakkinstian A, D'Este C, Attia J. A clinical decision rule to prioritize polysomnography in patients with suspected sleep apnea. Sleep. 2004;27(4):694-699.

4. Evans J, Skomro R, Driver H, et al. Sleep laboratory test referrals in Canada: sleep apnea rapid response survey. Can Respir J. 2014;21(1):e4-10

5. Alhadlaq A, Al-Maflehi N, Alzahrani S, AlAssiri A. Assessment of knowledge and attitude toward stem cells and their implications in dentistry among recent graduates of dental schools in Saudi Arabia. Saudi Dent J. 2019;31(1):66-75.

6. Al Agili DE. A needs assessment survey of dental public health graduate education in Saudi Arabia. Saudi Dent J. 2015;27(3):141148.

7. Al-Shalan TA. Dental education in Saudi Arabia: Areas of attention. Saudi Dent J. 2018;30(4): 271-272.

8. Al-Dlaigan YH, AlBarakati SF, Al-Habeeb F, Al-Hulaily M. Career characteristics and postgraduate education of female dentist graduates of the College of Dentistry at King Saud University, Saudi Arabia. Saudi Den J. 2012;24(1):29-34.

9. Alrahlah A. How effective the problem-based learning (PBL) in dental education. A critical review. Saudi Dent J.l 2016; 28(4):155161 .

10. Habib SR, Sattar K, Ahmad T, et al. An insightful evaluation of professionalism among dentistry students. Saudi Dent J. 2020.

11. Akinnusi M, Saliba R, El-Solh AA. Emerging therapies for obstructive sleep apnea. Lung 2012; 190(4):365-371.

13. Bahammam AS, Al-Rajeh MS, Al-Ibrahim FS, Arafah MA, Sharif MM. Prevalence of symptoms and risk of sleep apnea in middle-aged Saudi women in primary care. Saudi Med J. 2009;30(12):1572-1576.

14. BaHammam AS, Alrajeh MS, Al-Jahdali HH, BinSaeed AA Prevalence of symptoms and risk of sleep apnea in middle-aged Saudi males in primary care. Saudi Med J. 2008;29(3):423-426.

15. Elgezawi M, Hassan K, Alagl A, et al. Complexity of comprehensive care treatments in undergraduate dental programs: The benefits of observing and assisting experienced faculty members. Saudi Dent J. 2017;29(4):161-166.

16. Almeneessier AS, BaHammam AS. Sleep Medicine in Saudi Arabia J Clin Sleep Med. 2017;13(4): 641-645.

17. Aarab G, Lobbezoo F. Dental Sleep Medicine redefined. Sleep Breath. 2018;22(4):1233.

18. Al-Hussyeen AJA. Factors affecting utilization of dental health services and satisfaction among adolescent females in Riyadh City. Saudi Dent J. 2010;22(1):19-25.

19. Simmons MS, Pullinger A. Education in sleep disorders in US dental schools DDS programs. Sleep Breath. 2012;16(2):383-392.

Wali SO, Abalkhail B, Krayem A. Prevalence and risk factors of obstructive sleep apnea syndrome in a Saudi Arabian population. Ann Thorac Med. 2017;12(2): 88-94.

21. BaHammam A, Al-Jahdali H, AlHarbi A, AlOtaibi G, Asiri S, AlSayegh A. Saudi regulations for the accreditation of sleep medicine physicians and technologists. Ann Thorac Med. 2013;8(1):3-7. 

Arabia: A critical step toward quality outcomes. Ann Thorac Med. 2013;8(1): 1-2.

23. Bahammam AS. Sleep medicine in Saudi Arabia: Current problems and future challenges. Ann Thorac Med. 2011;6(1):3-10.

24. Talaat W, AlRozzi B, Kawas SA. Sleep medicine education and knowledge among undergraduate dental students in Middle East universities. Cranio. 2016; 34(3):163-168.

Al-Dlaigan YH, Al-Sadhan Re, Al-Ghamdi M, Al-Shahrani A, AlShahrani M. Postgraduate specialties interest, career choices and qualifications earned by male dentists graduated from King Saud University. Saudi Dent J. 2011;23(2):81-86.

Al-Sudani D, Al-Abbas F, Al-Bannawi Z, Al-Ramadhan A. Professional attitudes and behaviors acquired during undergraduate education in the College of Dentistry, King Saud University. Saudi Dentl J. 2013;25(2):69-74.

Saleem AH, Al Rashed FA, Alkharboush GA, et al. Primary care physicians' knowledge of sleep medicine and barriers to transfer of patients with sleep disorders. A cross-sectional study. Saudi Med J. 2017;38(5):553-559.

Quan SF, Schmidt-Nowara W. The role of dentists in the diagnosis and treatment of obstructive sleep apnea: consensus and controversy. J Clin Sleep Med. 2017;13(10):1117-1119.

29. Ramar K, Dort LC, Katz SG, et al. Clinical Practice Guideline for the Treatment of Obstructive Sleep Apnea and Snoring with Oral Appliance Therapy: An Update for 2015. J Clin Sleep Med. 2015; 11(7):773-827.

30. Flores-Mir C. Dentistry and Obstructive Sleep Apnea. J Clin Sleep Med. 2016;12(9):1213-1214.

31. Schwartz M, Acosta L, Hung YL, Padilla M, Enciso R. Effects of
CPAP and mandibular advancement device treatment in obstructive sleep apnea patients: a systematic review and meta-analysis. Sleep Breath. 2018;22(3):555-68

32. Van Haesendonck G, Dieltjens M, Kastoer C, et al. Cardiovascular Benefits of Oral Appliance Therapy in Obstructive Sleep Apnea: A Systematic Review; 2015.

33. Sutherland K, Vanderveken OM, Tsuda H, et al. Oral appliance treatment for obstructive sleep apnea: an update. J Clin Sleep Med. 2014;10(2):215-227.

\section{SUBMISSION \& CORRESPONDENCE INFORMATION}

\section{Submitted for publication December 23, 2019 \\ Submitted in final revised form June 5, 2020 \\ Accepted for publication June 15, 2020}

Address correspondence to: Dr. Nasser D. Alqahtani, Pediatric Dentistry and Orthodontics Department, College of Dentistry, King Saud University, Riyadh, Saudi Arabia. P.O. Box 231903 Riyadh, Zip code 11321; E-mail address: nasserdm@ksu.edu.sa

\section{DISCLOSURE STATEMENT}

The authors have no conflicts of interest to disclose. 